\title{
On Probabilistic Rationing Methods
}

\author{
Attila Tasnádi* \\ Department of Mathematics, Budapest University of Economic Sciences \\ and Public Administration, H-1093 Budapest, Fővám tér 8, Hungary ${ }^{\dagger}$
}

April 9, 2002.

Appeared in Mathematical Social Sciences 44(2002), p. 211-221, CElsevier Science S.A.

\begin{abstract}
Rationing occurs if the demand for a certain good exceeds its supply. In such situations a rationing method has to be specified in order to determine the allocation of the scarce good to the agents. Moulin (1999) introduced the notion of probabilistic rationing methods for the discrete framework. In this paper we establish a link between classical and probabilistic rationing methods. In particular, we assign to any given classical rationing method a probabilistic rationing method with minimal variance among those probabilistic rationing methods, which result in the same expected distributions as the given classical rationing method.
\end{abstract}

Keywords: Rationing; Indivisible units; Fair allocation.

JEL Classification Number: D45; D63.

\section{Introduction}

If for some good aggregate demand exceeds aggregate supply, then somehow aggregate supply has to be rationed to the individuals. Such situations may occur in various economic situations. For macroeconomics examples see for instance Clower (1965) and Benassy (1982), while for microeconomics examples consider Boyer and Moreaux (1989) and Gilbert and Klemperer (2000) among others. Furthermore, many interesting examples can be found in Young (1994) and Moulin (2000).

${ }^{*}$ I would like to thank this journal's Editor, an Associate Editor and an anonymous referee for valuable comments and suggestions, which led to substantial improvements in the paper. This research was done during the author's Bolyai János Research Fellowship provided by the Hungarian Academy of Sciences (MTA). All errors remain my own.

†Telephone: (+36 1) 4566782, E-mail: attila.tasnadi@math.bke.hu 
In the classical ${ }^{1}$ (continuous) model the scarce good is allocated deterministically and the supply of the scarce good, the agents' demands as well as the agents' shares are infinitely divisible; while in the discrete model the good comes in indivisible units but the allocation remains deterministic. Moulin (2000) characterized rationing methods in both the classical and the discrete model. Moulin (2000, Theorem 1) found that a rationing method satisfies a set of three reasonable axioms (consistency, upper composition and lower composition) if and only if it is a priority rule (that is the order in which the agents are served is predetermined and independent from the occurring rationing problem). In contrast to this negative result in the classical model the set of reasonable rationing methods is substantially larger (Moulin 2000, Theorem 2). Considering distributive justice the two models are behaving quite differently. Thus, it would be useful to establish a link between the two models.

The classical model can be linked with the discrete model if the indivisible units are sufficiently small since in that case the discrete model can be regarded as an approximation of the classical model. This paper follows another possibility by considering Moulin's (1999) probabilistic model in which the good comes in indivisible units (like in the discrete model) but the allocations are random. A connection will be established between the discrete framework and the classical framework by requiring that for discrete rationing problems a probabilistic rationing method equals a given classical rationing method in expected value. Particularly, we associate with a given classical rationing method a probabilistic rationing method with the same allocations in expected value and minimal variance for each individual. Therefore, we will call this method a minimal variance method associated with the given classical rationing method.

The classical proportional rationing method, which requires that the individual shares shall be determined in proportion to the individual demands, is one of the most natural methods to distribute the scarce good. Therefore, we will consider the minimal variance methods associated with the classical proportional method and we will refer to them as the fair remainder methods. Two axioms will be needed in order to provide our first axiomatic characterization of fair remainder methods (Proposition 2): proportional expected shares which is satisfied if the expected individual shares are in proportion to the individual demands, and proportion monotonicity which is fulfilled if larger proportions in demand lead to larger shares (in the sense of stochastic dominance). In addition, we present a second characterization (Proposition

\footnotetext{
${ }^{1}$ This model is called classical because it has received the most attention in the literature on rationing problems.
} 
3) which does not rely explicitly on the proportionality principle.

The rest of the paper is organized as follows. Section 2 describes the framework of our analysis. Section 3 introduces the minimal variance methods associated with a classical rationing method. Section 4 contains two axiomatic characterizations of the fair remainder rationing methods. Finally, Section 5 concludes our paper.

\section{The framework}

We denote by $\mathbb{N}$ the set of nonnegative integers and by $\mathbb{R}_{+}$the set of nonnegative real values. Let $\mathcal{N}$ be the finite set of potential agents. For any subset $N$ of $\mathcal{N}$, for any $x \in \mathbb{R}_{+}^{N}$ and any subset $M$ of $N$, we write $x_{M}=\sum_{i \in M} x_{i}$.

Let us denote by $N \subset \mathcal{N}$ the set of agents, by $t \in \mathbb{N}$ the quantity that has to be divided among agents in $N$ and by $x_{i} \in \mathbb{N}$ the demand of agent $i \in N$. Note that there is no need for rationing if $x_{N} \leq t$ since then everybody could receive their entire demanded quantity. Therefore, we will assume without loss of generality that $x_{N} \geq t$. We have to mention that we allow the case of $x_{N}=t$ because this simplifies the proof of Proposition 2. A discrete rationing problem is specified by the triple $\left(N, t,\left(x_{i}\right)_{i \in N}\right)$ for which we have $N \subset \mathcal{N}$, $t \in \mathbb{N}, x_{i} \in \mathbb{N}$ for all $i \in N$ and $x_{N} \geq t$.

In case of a discrete rationing problem $(N, t, x)$ we call a vector $y \in$ $\mathbb{R}_{+}^{N}$ an allocation if $y_{N}=t$ and $0 \leq y \leq x$. A classical rationing method $^{2} r$ assigns to any discrete rationing problem $(N, t, x)$ an allocation $y=r(N, t, x) \in \mathbb{R}_{+}^{N}$. A probabilistic rationing method assigns a probability distribution over integer valued allocations, i.e., if we denote by $\Omega_{N, t, x}:=\left\{\omega \in \mathbb{N}^{N} \mid \omega_{N}=t, 0 \leq \omega \leq x\right\}$ the set of possible integer valued allocations, then a probabilistic rationing method $\rho$ assigns to every discrete rationing problem $(N, t, x)$ a probability measure on the probability space $\left(\Omega_{N, t, x}, \mathcal{P}\left(\Omega_{N, t, x}\right)\right)$, where $\mathcal{P}\left(\Omega_{N, t, x}\right)$ denotes the power set of $\Omega_{N, t, x}$. For convenience, we will write $\rho_{N, t, x}$ instead of $\rho(N, t, x)$. Furthermore, for any agent $i \in N$ let us denote by $\rho_{N, t, x}^{i}$ the marginal distribution of agent $i$.

For reasons of convenience $E \rho_{N, t, x}$ stands for the expected value of a random variable corresponding to the probability measure $\rho_{N, t, x}$, i.e., $E \rho_{N, t, x}^{i}=\sum_{k=0}^{x_{i}} k \rho_{N, t, x}^{i}(k)$ for any $i \in N$. We will assign to any classical rationing method $r$ a probabilistic rationing method $\rho$ so that for any discrete rationing problem this allocates in expected value the same shares as $r$, that

\footnotetext{
${ }^{2}$ Classical rationing methods are usually defined for any continuous rationing problem (i.e., $t \in \mathbb{R}_{+}$and $x \in \mathbb{R}_{+}^{N}$ ), but with the exceptions of the second half of Section 4 we will only consider discrete rationing problems.
} 
is, $r(N, t, x)=E \rho_{N, t, x}$. We shall denote by $\mathcal{E}(r)$ the set of such probabilistic rationing methods.

Probabilistic rationing methods involve uncertainty. Thus, a risk averse individual prefers a rationing method with a smaller variance of the obtained amount to a rationing method with higher variance if both allocate the same amount to the individual in expected value. For any probabilistic rationing method, for any discrete rationing problem $(N, t, x)$ and for any individual $i \in N$ the individual variance of shares equals

$$
\operatorname{Var}\left(\rho_{N, t, x}^{i}\right)=\sum_{k=0}^{x_{i}}\left(k-E \rho_{N, t, x}^{i}\right)^{2} \rho_{N, t, x}^{i}(k) .
$$

\section{The minimal variance rationing methods}

In this section we associate a probabilistic rationing method with a classical rationing method and we determine how this associated method can be computed. Especially, this also ensures that $\mathcal{E}(r)$ is nonempty for any classical rationing method $r$.

For a given classical rationing method $r$ we define a probabilistic rationing method $\rho$ to be an associated minimal variance method if $\rho \in \mathcal{E}(r)$ and if for any discrete rationing problem $(N, t, x)$ we have

$$
\forall \mu \in \mathcal{E}(r): \forall i \in N: \operatorname{Var}\left(\rho_{N, t, x}^{i}\right) \leq \operatorname{Var}\left(\mu_{N, t, x}^{i}\right) .
$$

Next, we turn to the question how a minimal variance method associated with a classical rationing method $r$ can be determined. Take an arbitrarily fixed discrete rationing problem $(N, t, x)$. In step one, any agent $i \in N$ receives $y_{i}^{*}=\left\lfloor r_{i}(N, t, x)\right\rfloor$ units of the supply $t$, where $\lfloor a\rfloor$ stands for the largest integer not greater than $a \in \mathbb{R}$. After step one, the residual of agent $i \in N$, denoted by $u_{i}$, equals $u_{i}=r_{i}(N, t, x)-\left\lfloor r_{i}(N, t, x)\right\rfloor$. In step two, we have to distribute the remaining amount so that $r=E \rho$. We achieve this, for instance, if the individuals' marginal distributions equal

$$
\rho_{N, t, x}^{i}\left(y_{i}^{*}+1\right)=u_{i} \quad \text { and } \quad \rho_{N, t, x}^{i}\left(y_{i}^{*}\right)=1-u_{i} .
$$

The following theorem establishes that we can find a probabilistic rationing method satisfying (1) and thus, the marginal distributions in (1) determine indeed the minimal variance methods associated with $r$.

Theorem 1. A probabilistic rationing method $\rho \in \mathcal{E}(r)$, where $r$ is a given classical rationing method, is a minimal variance method associated with $r$ if and only if each agent's realized share differs by less than one from his 
expected share. In particular, the associated minimal variance methods are specified through the individual marginal distributions in (1).

Proof. We suppose for notational convenience that $N=\{1, \ldots, n\}$. Let us denote by $M$ the set of those agents, who do not obtain an integer share in case of applying $r$, that is, $M=\left\{i \in N \mid y_{i}^{*}<r_{i}(N, t, x)\right\}$. Observe that we are done if set $M$ is empty or if equivalently $y_{N}^{*}=t$. Therefore, in what follows we assume that $y_{N}^{*}<t$. For notational convenience we suppose that $M=\{1, \ldots, m\}$. We have to specify $\rho$ appropriately. The support of $\rho$ is subset $\Omega_{N, t, x}^{*}=$

$$
\left\{\omega^{*} \in \mathbb{N}^{N} \mid \omega_{N}^{*}=t, \forall i \in M: \omega_{i}^{*} \in\left\{y_{i}^{*}, y_{i}^{*}+1\right\} \text { and } \forall i \in N \backslash M: \omega_{i}^{*}=y_{i}^{*}\right\} \text {, }
$$

of $\Omega_{N, t, x}$. The cardinality of set $\Omega_{N, t, x}^{*}$ equals $q:=\left(\begin{array}{c}m \\ t-y_{N}^{*}\end{array}\right)$. Note that $m>$ $t-y_{N}^{*}$ and thus $q \geq m$. Let the sequence $\left(a_{i}\right)_{i=1}^{q}$ be an enumeration of the elements of set $\Omega_{N, t, x}^{*}-y^{*}:=\left\{z \in \mathbb{N}^{N} \mid \exists \omega^{*} \in \Omega_{N, t, x}^{*}: z=\omega^{*}-y^{*}\right\}$. We shall denote by $a_{i j} \in\{0,1\}$ the amount distributed to agent $j \in N$ in step two by $a_{i}(i \in\{1, \ldots, q\})$. Clearly, $a_{i j}=0$ for any $j \in N \backslash M$. Since (1) shall hold we obtain the following linear equation system with variables $\alpha_{i} \geq 0$ $(i=1, \ldots, q)$

$$
\sum_{i=1}^{q} \alpha_{i} a_{i j}=u_{j}
$$

for all $j \in M$. Observe that $\sum_{j=1}^{m} a_{i j}=\sum_{j=1}^{n} a_{i j}=t-y_{N}^{*}$ for all $i=1, \ldots, q$. We can verify by summing the $m$ equations in (2) that $\sum_{i=1}^{q} \alpha_{i}=1$ holds true. The existence of a nonnegative solution of (2) follows from Lemma 4, which we state and prove in the Appendix. In fact the proof of Lemma 4 is constructive so that we can find $\rho$ for instance by applying the simplex method. Finally, it follows immediately from (1) that $\rho$ is a minimal variance method associated with $r$.

A nonnegative solution of (2) determines the required probability measure $\rho_{N, t, x}$ needed to specify the associated minimal variance method. Problem (2) may have multiple nonnegative solutions. However, any solution results in the same one dimensional marginal distributions and satisfies $r=E \rho$. Hence, we can specify an associated minimal variance method based on an arbitrary nonnegative solution of (2).

\section{The fair remainder rationing methods}

Among the classical rationing methods the proportional method, given by $\operatorname{pro}(N, t, x):=\left(t / x_{N}\right) x$ whenever $x_{N}>0$ and by $\operatorname{pro}_{i}(N, t, x):=0$ for all 
$i \in N$ whenever $x_{N}=0$, is of special interest. Probabilistic rationing methods in $\mathcal{E}($ pro $)$ can be regarded as fair, in some sense, because they satisfy by definition:

Axiom 1. Proportional expected shares: For any discrete rationing problem $(N, t, x)$ we have $E \rho_{N, t, x}=\operatorname{pro}(N, t, x)$.

We call the minimal variance methods associated with the classical proportional method the fair remainder methods.

In this section we present two characterizations of the fair remainder methods. But before we can state the first one we have to introduce the axiom of proportion monotonicity, which requires that for any agent a larger proportion in demand leads to higher shares, in the sense of stochastic dominance. The stochastic dominance relation, denoted by $\leq$, between two probability measures $\mu$ and $\nu$ on $(\{0,1, \ldots, n\}, \mathcal{P}(\{0,1, \ldots, n\}))$ can be defined in the following way:

$$
\mu \leq \nu \Leftrightarrow \forall k \in\{0,1, \ldots, n\}: \mu(\{k, k+1, \ldots, n\}) \leq \nu(\{k, k+1, \ldots, n\}) .
$$

Axiom 2. Proportion monotonicity: For any pair of discrete rationing problems $(N, t, x)$ and $(N, t, y)$ such that $x_{N}>0$ and $y_{N}>0$ we have

$$
\frac{x_{i}}{x_{N}} \leq \frac{y_{i}}{y_{N}} \Rightarrow \rho_{N, t, x}^{i} \leq \rho_{N, t, y}^{i}
$$

for any $i \in N$.

Now, we can give our first characterization of the fair remainder methods.

Proposition 2. A probabilistic rationing method is a fair remainder method if and only if it satisfies proportion monotonicity and proportional expected shares.

Proof. It follows easily from its definition and Theorem 1 that a fair remainder method meets proportion monotonicity and proportional expected shares.

To demonstrate the converse statement take an arbitrary discrete rationing problem $(N, t, x)$. Observe that there is nothing to prove if $t=0$ or $x_{N}=x_{i}$ for a certain $i \in N$, since in these two cases any probabilistic rationing method results in the same deterministic allocation. Therefore, in what follows we can assume that $t>0$ and $x_{N}>x_{i}$ for all $i \in N$. Note that we have only to show that any agent $i \in N$ obtains with positive probability either an amount of $\left\lfloor t x_{i} / x_{N}\right\rfloor$ or $\left\lfloor t x_{i} / x_{N}\right\rfloor+1$ since then the converse statement is implied by proportional expected shares. Pick an agent $i \in N$. 
We select demand vectors $y$ and $z$ so that $y_{i}=\left\lfloor t x_{i} / x_{N}\right\rfloor, y_{N \backslash i}=t-y_{i}$, $z_{i}=y_{i}+1, z_{N \backslash i}=t-z_{i}$ and $y_{j}, z_{j} \in \mathbb{N}$ for all $j \neq i$. Clearly, by $y_{i} \leq t x_{i} / x_{N}$ and $y_{N \backslash i}=t-y_{i}$ it follows that $y_{i} / y_{N} \leq x_{i} / x_{N}$. Similarly, by $z_{i} \geq t x_{i} / x_{N}$ and $z_{N \backslash i}=t-z_{i}$ it follows that $x_{i} / x_{N} \leq z_{i} / z_{N}$. For rationing problem $(N, t, y)$ we have $\Omega_{N, t, y}=\{y\}$ and thus, $y$ is the only possible allocation by the definition of probabilistic rationing methods. But then by proportion monotonicity it follows for rationing problem $(N, t, x)$ that agent $i$ obtains less than $y_{i}$ units with zero probability. Again from the definition of probabilisitc rationing methods it follows for rationing problem $(N, t, z)$ that the assigned quantities equal $z$. Therefore, by proportion monotonicity we conclude that agent $i$ obtains more than $z_{i}$ units with zero probability in rationing problem $(N, t, x)$, which completes the proof of our proposition.

Now, we argue for the sustainability of the two requirements in Proposition 2. It does not seem to offend us that a larger share in demand shall lead to a larger share in allocation. Hence, we would accept proportion monotonicity. Furthermore, proportional expected shares can be regarded as a requirement of fairness.

We have to verify that the statement in Proposition 2 is tight in the sense that dropping any of the two axioms will allow for other probabilistic rationing methods besides the fair remainder methods. The proportional probabilistic rationing method $^{3}$ is an example for rationing methods fulfilling proportional expected shares but violating proportion monotonicity. The latter statement can be verified through comparing rationing problems $(\{1,2,3\}, 3,(1,1,1))$ and $(\{1,2,3\}, 3,(1,2,3))$. Although in the second one agent 3 has a larger share in demand, he receives 0 units with positive probability, while in the first one he never receives 0 units. For a probabilistic rationing method violating only proportional expected shares we modify a fair remainder method slightly. For discrete rationing problems of type $\left(\{1,2\}, 1,\left(x_{1}, x_{2}\right)\right)$ let $\rho_{\{1,2\}, 1,\left(x_{1}, x_{2}\right)}(1,0)=1$ if $x_{1} \geq 1$, and let $\rho_{\{1,2\}, 1,\left(x_{1}, x_{2}\right)}(0,1)=1$ if $x_{1}=0$. For any other discrete rationing problem the probabilistic rationing method is defined in the fair remainder manner. It can be easily checked that this rationing method meets proportion monotonicity, but violates proportional expected shares.

We can characterize the fair remainder methods easily in other ways without relying on the proportionality principle by applying already existing characterizations of the classical proportional method (see for instance Banker,

\footnotetext{
${ }^{3}$ Moulin (1999) defines and characterizes the proportional probabilistic rationing method. Briefly, this method can be implemented, for example, through successive random rounds, where in each round the probabilities of obtaining the next unit are in proportion to the unsatisfied demands.
} 
1981; O’Neill, 1982; Moulin, 1987; Chun, 1988; and Young, 1988). We will apply the simplest of the four characterizations appearing in Moulin (2001) Theorem 1.1. Therefore, we will introduce two other properties. The first one requires that each agent's possible shares differ by at most one unit.

Axiom 3. A probabilistic rationing method $\rho$ is almost deterministic if for any discrete rationing problem $(N, t, x)$ and any $i \in N$ there exists a $y_{i} \in$ $\left\{0,1, \ldots, x_{i}-1\right\}$ such that $\rho_{N, t, x}^{i}\left(\left\{y_{i}, y_{i}+1\right\}\right)=1$.

To apply existing characterizations of the classical proportional method we have to extend our framework. In Section 2 we only required that classical rationing methods are defined for discrete rationing problems because this was appropriate for our analysis so far. However, rationing methods can also be defined for problems in which the demands $x_{i}(i \in N)$ and the supply $t$ are nonnegative real values. A rationing problem $\left(N, t,\left(x_{i}\right)_{i \in N}\right)$ is said to be continuous if $N \subset \mathcal{N}, t \in \mathbb{R}_{+}, x_{i} \in \mathbb{R}_{+}$for all $i \in N$ and $x_{N} \geq t$. Clearly, the proportional method pro is defined for any continuous rationing problem. For any given probabilistic rationing method $\rho$ we can extend the classical rationing method $E \rho$, which assigns to each discrete rationing problem an allocation, to the set of continuous rationing problems. In particular, $F$ is an extension of $E \rho$ to the set of continuous rationing problems, henceforth briefly an extension of $E \rho$, if $F$ is defined, satisfies $0 \leq F(N, t, x) \leq x$ and $F_{N}(N, t, x)=t$ for any continuous rationing problem, and $F(N, t, x)=$ $E \rho_{N, t, x}$ for any discrete rationing problem. Of course, there are many possible extensions of $E \rho$.

Our last property requires for an extension $F$ of $E \rho$ that transfers of demands across a subset of agents do not affect the expected total share of this coalition. ${ }^{4}$

Axiom 4. Let $\rho$ be a probabilistic rationing method. Then an extension $F$ of $E \rho$ fulfills no advantageous reallocation if $F_{M}(N, t, x)=F_{M}\left(N, t, x^{\prime}\right)$ for all pairs of continuous rationing problems $(N, t, x),\left(N, t, x^{\prime}\right)$ and all $M \subset N$ such that $x_{M}=x_{M}^{\prime}$ and $x_{i}=x_{i}^{\prime}$ for all $i \in N \backslash M$.

Now we are ready to state our second characterization of the fair remainder methods, which does not refer explicitly to the proportionality principle.

Proposition 3. Suppose $N$ contains at least three agents. Then a probabilistic rationing method $\rho$ is a fair remainder method if and only if it is almost deterministic and there exists an extension $F$ of E $\rho$ satisfying no expected advantageous reallocation.

\footnotetext{
${ }^{4}$ Moulin (1987) and Chun (1988) used the axiom of no advantageous reallocation in characterizations of the classical proportional method.
} 
Proof. By Theorem 1 the fair remainder rationing methods are exactly those which are almost deterministic and satisfy proportional expected shares. Thus, if $\rho$ is a fair remainder method, then pro, which is defined for any continuous rationing problem, is an extension of $E \rho$. Since pro statisfies no advantageous rellocation (see Moulin, 2001 Theorem 1.1), F := pro is an appropriate extension of $E \rho$.

To establish the converse statement suppose that $\rho$ is almost deterministic and $F$ is an extension of $E \rho$ satisfying no advantageous reallocation. Then it follows from Moulin (2001) Theorem 1.1 that $F=$ pro. Hence, $\rho$ fulfills proportional expected shares. Finally, applying that $\rho$ is almost deterministic, we derive that $\rho$ has to be a fair remainder method.

Finally, let us remark that by applying other characterizations of the classical proportional rationing method further characterizations of the fair remainder methods can be obtained in an analogous way to Proposition 3. In particular, to derive another characterization of the fair remainder methods one needs Axiom 3 and the existence of an extension $F$ of $E \rho$ satisfying the Axioms appearing in the applied characterization of the classical proportional rationing method.

\section{Conclusions}

In this paper we have established a connection between classical rationing methods and probabilistic rationing methods. In particular, we defined a correspondence from the set of classical rationing methods to the set of probabilistic rationing methods (by requiring expected allocations to equal the allocations resulting from a given classical rationing method and selecting from such probabilistic methods those with minimal variance). In the classical (continuous) model the proportional, the uniform gains ${ }^{5}$ and the uniform losses methods ${ }^{6}$ are of special importance because of their axiomatic properties (see Moulin, 2000). Moulin and Stong (2000) found that in the probabilistic model the proportional probabilistic, the fair queuing and the fair queuing* methods play the same roles respectively as the previously listed three classical methods. ${ }^{7}$

\footnotetext{
${ }^{5}$ Specified by $u g_{i}(N, t, x):=\min \left\{\lambda, x_{i}\right\}$, where $\sum_{i \in N} \min \left\{\lambda, x_{i}\right\}=t$.

${ }^{6}$ Specified by $u l_{i}(N, t, x):=\max \left\{x_{i}-\mu, 0\right\}$, where $\sum_{i \in N} \max \left\{x_{i}-\mu, 0\right\}=t$.

${ }^{7}$ The fair queuing method allocates the insufficient supply in successive round-robin fashion so that in each round the consumers, who still have unsatisfied demand, receive an additional unit in a random order while there are remaining units left. The fair queuing* method allocates losses in an analogous way.
} 
It can be easily checked that the fair queuing method is a minimal variance method associated with $u g$, while the fair queuing* method is a minimal variance method associated with $u l$. Thus, from a point of view other than Moulin's and Stong's (2000), we can confirm that the fair queuing method corresponds to the uniform gain method, while the fair queuing* method corresponds to the uniform losses method. However, our correspondence points out a different probabilistic rationing method for the proportional method, which we called fair remainder. Following from its definition a fair remainder method has the smallest level of uncertainty among those rationing methods that satisfy proportional expected shares. We characterized the fair remainder methods, first, by proportion monotonicity and proportional expected shares. In the presence of at least three agents our second characterization shows that the fair remainder methods are exactly those methods which are almost deterministic and which satisfy that all subsets of agents cannot improve their expected total share by redistributing their demands.

\section{Appendix}

In the appendix we state and prove a Lemma, which guarantees the existence of a nonnegative solution of problem (2). Take two positive integers $k$ and $m$ so that $k<m$. Let $q:=\left(\begin{array}{c}m \\ k\end{array}\right), M:=\{1, \ldots, m\}, Q:=\{1, \ldots, q\}$ and let $\mathcal{M}_{m, k}$ denote the set of those $q \times m$ matrixes that contain only zeros and ones so that they have $q$ different rows with each row containing exactly $k$ ones. This means that an $A \in \mathcal{M}_{m, k}$ contains every possible distribution of $k$ ones in its rows. Formally, $\mathcal{M}_{m, k}:=$

$$
\left\{A \in\{0,1\}^{q \times m} \mid \forall i \in Q: \sum_{j=1}^{m} a_{i j}=k ; \forall i, j \in Q, i \neq j: \exists l \in M: a_{i l} \neq a_{j l}\right\} .
$$

We are looking for a nonnegative solution $y \in \mathbb{R}^{q}$ of the linear equation system

$$
y A=b,
$$

for a given $A \in \mathcal{M}_{m, k}$ and $b \in(0,1)^{m}$ for which $\sum_{i=1}^{m} b_{i}=k$.

Lemma 4. Problem (3) has a nonnegative solution.

Proof. The matrix $A=\left(a_{i j}\right)_{q \times m}$ has $m$ linearly independent rows because any of the unit vectors $e_{j} \in \mathbb{R}^{m}$ can be obtained by the linear combination of rows $a_{i}=\left(a_{i 1}, \ldots, a_{i m}\right)$. To verify this check that

$$
e_{j}=\frac{\sum_{i=1}^{q} a_{i}}{\left(\begin{array}{c}
m-1 \\
k-1
\end{array}\right)}-\frac{\sum_{i \in I_{j}} a_{i}}{\left(\begin{array}{c}
m-2 \\
k-1
\end{array}\right)}
$$


where $I_{j}=\left\{i \in Q \mid a_{i j}=0\right\}$ and $j \in M$. Thus, we know that (3) has a solution, but we do not know whether it has a nonnegative solution. Therefore, we will consider the following linear programming problem:

$$
\begin{aligned}
y A & \leq b \\
y & \geq \mathbf{0}_{q} \\
\sum_{i=1}^{q} y_{i} & \rightarrow \max
\end{aligned}
$$

where $\mathbf{0}_{q}=(0, \ldots, 0) \in \mathbb{R}^{q}$. From $y A \leq b$ it follows that $\sum_{i=1}^{q} y_{i} \leq 1$. Hence (4) has an optimal value. If the maximum equals 1 , then the optimal solution of (4) is also a solution to (3). To verify that indeed the maximum equals 1 we will investigate the dual problem of (4)

$$
\begin{aligned}
A x & \geq \mathbf{1}_{q} \\
x & \geq \mathbf{0}_{m} \\
b x & \rightarrow \text { min }
\end{aligned}
$$

where $x \in \mathbb{R}^{m}$ and $\mathbf{1}_{q}=(1, \ldots, 1) \in \mathbb{R}^{q}$. Since (5) has an optimal value, we must find an optimal point among the extreme points of the set $S=$ $\left\{x \in \mathbb{R}^{m} \mid A x \geq \mathbf{1}_{q}, x \geq \mathbf{0}_{m}\right\}$. It can be checked that the set of its extreme points equals $S_{e}=$

$$
\left\{x \in S|\exists I \subset M:| I \mid<k, x_{i}=\frac{1}{k-|I|} \text { for } i \in M \backslash I, x_{i}=0 \text { for } i \in I\right\} .
$$

To find an optimal point we have to evaluate the object function of the dual problem (5) above set $S_{e}$. For $x^{*}=\mathbf{1}_{m} / k \in S_{e}$ we obtain $b x^{*}=1$. For any $x \in S_{e} \backslash\left\{x^{*}\right\}$, with a corresponding set of indices $I \subset M$ so that $0<|I|<k$, the following inequality holds true

$$
\begin{aligned}
b x & =\frac{1}{k-|I|} \sum_{i \in M \backslash I} b_{i}=\frac{1}{k-|I|}\left(\sum_{i \in M} b_{i}-\sum_{i \in I} b_{i}\right)> \\
& >\frac{1}{k-|I|}\left(\sum_{i \in M} b_{i}-\sum_{i \in I} 1\right)=\frac{1}{k-|I|}(k-|I|)=1=b x^{*}
\end{aligned}
$$

and therefore we conclude that $x^{*}$ is the unique optimal point of (5), which in turn implies that the maximum of problem (4) equals 1.

The solution of problem (4) and therefore a nonnegative solution of (3) can be determined for instance by the simplex method. 


\section{References}

[1] Banker, R., 1981. Equity Considerations in Traditional Full Cost Allocation Practices: An Axiomatic Perspective. In: Moriarty, S., (Ed.), Joint Cost Allocations. University of Oklahoma, Norman, pp. 110-130.

[2] Benassy, J.-P., 1982. The Economics of Market Disequilibrium. Academic Press, New York.

[3] Boyer, M., Moreaux, M., 1989. Endogenous Rationing in a Differentiated Duopoly. International Economic Review 30, 877-888.

[4] Chun, Y., 1988. The Proportional Solution for Rights Problems. Mathematical Social Sciences 15, 231-246.

[5] Clower, R.W., 1965. The Keynesian counter-revolution: A theoretical appraisal. In: Hahn, F.H., Brechling, F.P.R., (Eds.), The theory of interest rates. Macmillan, London, pp. 103-125.

[6] Gilbert, R.J., Klemperer, P., 2000. An Equilibrium Theory of Rationing. RAND Journal of Economics 31, 1-21.

[7] Moulin, H., 1987. Equal or Proportional Division of a Surplus, and Other Methods. International Journal of Game Theory 16, 161-186.

[8] Moulin, H., 1999. The Proportional Random Allocation Of Indivisible Units. Social Choice and Welfare, forthcoming.

[9] Moulin, H., 2000. Priority Rules and Other Asymmetric Rationing Methods. Econometrica 68, 643-684.

[10] Moulin, H., 2001. Axiomatic Cost and Surplus-Sharing. In: Arrow, K.J., Sen, A.K., Suzumura, K., (Eds.), Handbook of Social Choice and Welfare, Volume 1. North-Holland, Amsterdam, forthcoming.

[11] Moulin, H., Stong, R., 2000. Fair Queuing and Other Probabilistic Allocation Methods. Mathematics of Operations Research, forthcoming.

[12] O'Neill, B., 1982. A Problem of Rights Arbitration from the Talmud. Mathematical Social Sciences 2, 345-371.

[13] Young, H.P., 1988. Distributive Justice in Taxation. Journal of Economic Theory 44, 321-335.

[14] Young, H.P., 1994. Equity: in Theory and Practice. Princeton University Press, Princeton. 